\title{
Development of multivalent nanobodies blocking SARS-CoV-2 infection by targeting RBD of spike protein
}

\author{
Qizhong $\mathrm{Lu}^{1+} \mathbb{0}$, Zongliang Zhang ${ }^{1 \dagger}$, Hexian $\mathrm{Li}^{1 \dagger}$, Kunhong Zhong ${ }^{1}$, Qin Zhao ${ }^{2}$, Zeng Wang ${ }^{1}, Z_{\text {higuo Wu}}$, \\ Donghui Yang ${ }^{3}$, Shuang Sun ${ }^{1}$, Nian Yang ${ }^{1}$, Meijun Zheng ${ }^{4}$, Qiang Chen' ${ }^{1}$, Cheng Long ${ }^{5}$, Wenhao Guo', \\ Hui Yang ${ }^{4}$, Chunlai $\mathrm{Ni}^{1 *}$ and Aiping Tong ${ }^{1 *}$
}

\begin{abstract}
Background: The outbreak and pandemic of coronavirus SARS-CoV-2 caused significant threaten to global public health and economic consequences. It is extremely urgent that global people must take actions to develop safe and effective preventions and therapeutics. Nanobodies, which are derived from single-chain camelid antibodies, had shown antiviral properties in various challenge viruses. In this study, multivalent nanobodies with high affinity blocking SARS-CoV-2 spike interaction with ACE2 protein were developed.

Results: Totally, four specific nanobodies against spike protein and its RBD domain were screened from a naïve VHH library. Among them, Nb91-hFc and Nb3-hFc demonstrated antiviral activity by neutralizing spike pseudotyped viruses in vitro. Subsequently, multivalent nanobodies were constructed to improve the neutralizing capacity. As a result, heterodimer nanobody Nb91-Nb3-hFc exhibited the strongest RBD-binding affinity and neutralizing ability against SARS-CoV-2 pseudoviruses with an $\mathrm{IC}_{50}$ value at approximately $1.54 \mathrm{nM}$.

Conclusions: The present study indicated that naive VHH library could be used as a potential resource for rapid acquisition and exploitation of antiviral nanobodies. Heterodimer nanobody Nb91-Nb3-hFc may serve as a potential therapeutic agent for the treatment of COVID-19.
\end{abstract}

Keywords: SARS-CoV-2, Nanobody, Spike, RBD, Pseudovirus neutralization

\section{Background}

Coronavirus disease 2019 (COVID-19) is caused by infection of severe acute respiratory syndrome coronavirus 2 (SARS-CoV-2) $[1,2]$. It has spread global and had been announced by World Health Organization (WHO) in March 2020 as the first coronavirus severe pandemic in the history of humanity [3, 4]. More than 83.92 million individuals have been infected and caused about

\footnotetext{
*Correspondence: niecl1022@scu.edu.cn; aipingtong@scu.edu.cn ${ }^{\dagger}$ Qizhong Lu, Zongliang Zhang and Hexian Li contributed equally to thiswork and considered as co-first authors

1 State Key Laboratory of Biotherapy and Cancer Center, West China Hospital, Sichuan University, Chengdu 610041, China

Full list of author information is available at the end of the article
}

1.82 million people deaths globally (as of January 2, 2021, source: Johns Hopkins University), and the number is still increasing. It is extremely urgent that global people must take actions to develop safe and effective preventions and therapeutics.

SARS-CoV-2 is an enveloped virus that belongs to the family Coronaviridae, the subfamily Orthocoronaviri$d a e$ and genus $\beta$-coronavirus [5]. The virus genome is a positive-sense, single-stranded RNA with a full length of $30.0 \mathrm{~kb}$, which is $96.2 \%$ identical to a bat CoV RaTG13, whereas it shares $79.6 \%$ identity to SARS-CoV $[5,6]$. Its genome consists of six functional open-reading frames (ORFs), which encoded replicase (ORF1a/ORF1b), membrane $(\mathrm{M})$, spike glycoprotein $(\mathrm{S})$, envelope $(\mathrm{E})$ and 
nucleocapsid $(\mathrm{N})$, most of the proteins encoded by SARSCoV-2 are similar with SARS-CoV $[7,8]$. S protein can form a homotrimer complex and extrude from envelope to form the coronal in terms of morphological structure. It can be structurally or functionally divided into two subunits, called S1 and S2. S1 subunit contains the receptor-binding domain (RBD), which binds to the extracellular domain of receptor angiotensin converting enzyme 2 (ACE2) and mediate the virus entry into host cells, while the $\mathrm{S} 2$ subunit is necessary for membrane fusion [9-12]. Based on the characteristics of SARS-CoV-2 RBD immunogen could induce neutralizing antibody in animals and is necessary for virus infection in host cells, thus, it can be used as a good target for the development of neutralizing antibodies $[13,14]$.

Heavy-chain only antibodies (hcAbs) derived from camelids or sharks that devoid of light chains and lack $\mathrm{CH} 1$, nevertheless have an extensive antigen-binding repertoire, its variable domain was named $\mathrm{VHH}$ or nanobody $(\mathrm{Nb})(15 \mathrm{kDa})$ [15-18]. Nanobody offer advantages including high affinity and specificity, smaller size (1/10th the size of conventional monoclonal antibodies), thermostability, low immunogenicity, and excellent tissue penetration-characteristics that are widely applied in oncotherapy, diagnosis and monitoring of disease, and prevent virus infection [19-24]. For example, numerous studies about Nbs antiviral activity for various challenging viruses have been reported, including MERS, HIV, HCV, IAV and SFTSV [24-27]. While many candidates are in preclinical development and several antibodies (VIR-7831, LY-CoV016, BGB-DXP593, and CT-P59) have entered late-stage clinical trials, two neutralizing antibodies, Lilly's LY-Cov555 and Regeneron's REGN-COV2, have received FDA emergency use authorization for the treatment of COVID-19. For nanobodies, most of them are in preclinical trials. For example, Twist Bioscience Corporation recently announced two nanobodies, TB202-3 and TB202-63, protect against weight loss, a key indicator of disease severity, at the dose of $1 \mathrm{mg} / \mathrm{kg}$ in a preclinical hamster challenge model.

Here, several nanobodies directed to spike protein and its RBD domain with high affinity were obtained after multiple rounds of enrichment from a naïve $\mathrm{VHH}$ library (Scheme 1a). Based on the production platform of the nanobody-hFc, S and RBD protein specific nanobodies were expressed (Scheme 1a). To determinate the neutralizing activity of specific nanobodies, the SARS-CoV-2 spike pseudotyped lentivirus were produced firstly using HEK293T cells (Scheme 1b). Neutralizing nanobodies could significantly inhibit SARS-CoV-2 pseudoviruses infection in host HEK293T-ACE2 cells through blocking spike protein interaction with ACE2 by targeting RBD (Scheme 1c).

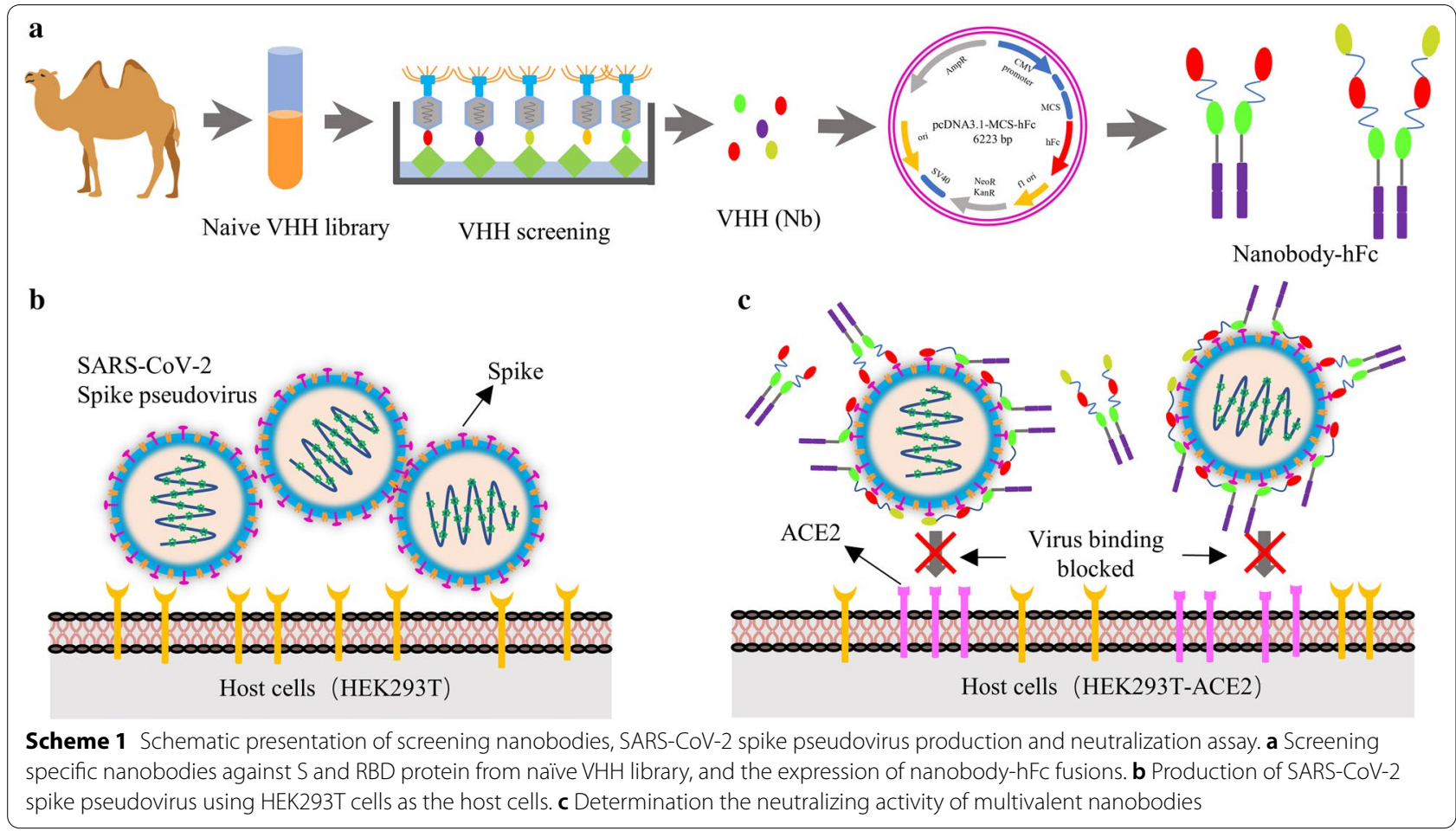


We believe that nanobodies may serve as a potential agent for prevention and therapy of COVID-19.

\section{Materials and methods \\ Cell lines and vectors}

HEK293T cell lines were purchased from ATCC and cultured in Dulbecco's Modified Eagle's Medium (Gibco, USA) containing $10 \%$ fetal bovine serum (FBS, BI, USA) at $37{ }^{\circ} \mathrm{C}$ in $5 \% \mathrm{CO}_{2}$. Spodoptera fruited (sf9) cells were maintained in the SIM SF medium (Sino Biological, Beijing, China). All cell lines have been tested negative for contamination with mycoplasma. HEK293T cells were used to construct HEK293T-ACE2 stable cell lines, express recombinant nanobodies, and produce SARSCoV-2 pseudovirus of the novel coronavirus; sf9 cells were applied to express spike and RBD protein of SARS$\mathrm{CoV}-2$. The pMECS vector was used to construct phage display library. The pcDNA3.1 vector (V790-20, invitrogen, USA) was used for eukaryotic expression of the nanobodies. pLenti-EGFP-luciferase expressing plasmid and gag/pol plasmid were used to prepare SARS-CoV-2 pseudoviruses.

\section{Gene cloning, protein expression and purification of recombinant SARS-CoV-2 proteins}

The spike and RBD protein of SARS-CoV-2 were expressed using the Bac-to-Bac baculovirus expression system (Invitrogen) as previously described [14]. Briefly, a gp67 signal peptide sequence [28] was inserted into pFastBac1 vector between $B a m H I$ and EcoRI restriction sites, and then the coding sequence (codon optimized for insect cells) of spike ECD (extracellular domain, 16-1213 aa) and RBD (319-545 aa) of SARS-CoV-2 Wuhan-Hu-1 isolate (Genbank accession number MN908947) were followed by the gp67 signal peptide. In addition, $8 \times$ His tag was fused to C-terminal to profit protein purification. The bacmid was transfected into sf9 cells with LipoInsect Transfection regent (Beyotime Biotechnology, Jiangsu, China) according to the manufacture's instruction for recombinant baculovirus package and protein expression. Subsequently, the culture supernatant that containing $S$ and RBD protein were harvested after $72 \mathrm{~h}$ transfection and purified using Ni-NTA 6FF Agarose (SMART, Changzhou, China), respectively. The expression and purity of recombinant were analyzed by SDS-PAGE.

\section{Construction of naïve VHH library}

Total $200 \mathrm{~mL}$ Bactrian camel blood samples (containing 50 camels) were collected from Jinchang city, Gansu province in China. The peripheral blood lymphocytes (PBLs) were extracted by Leucosep ${ }^{\circledR}$ tubes (Greiner BioOne, Germany) for naïve library construction. Total RNA was extracted and used to synthesise the cDNA. Next, the $\mathrm{VHH}$ genes were amplified by nest-PCR according to previous description [29-31], and then cloned into phagemid pMECS vector. The recombinant phagemids were electro-transformed into freshly competent E. coli TG1 Electrocompetent cells. Cells were cultured on LB agar plates that containing ampicillin and D-glucose overnight at $37{ }^{\circ} \mathrm{C}$. On the second day, the cells were scraped and stored at $-80{ }^{\circ} \mathrm{C}$ at LB medium, and then the positive rate of the constructed library was determined according to previous description [32]. Finally, 36 clones were randomly selected for sequencing to analyze the library's diversity.

\section{Screening and identification specific nanobodies against SARS-CoV-2 spike and RBD protein}

To select the spike and RBD nanobodies, four rounds of phage rescue and screening were performed as described previously [30, 31, 33]. Briefly, purified S and RBD protein were immobilized in microtiter plate (Nunc, Thermo). For each round an uncoated well was used as a negative control. The wells were washed with PBS'T buffer (PBS with $0.05 \%$ Tween $20(\mathrm{v} / \mathrm{v}))$ and then were blocked with blocking buffer (PBS'T containing $5 \%(\mathrm{w} / \mathrm{v})$ skimmed milk) at $37{ }^{\circ} \mathrm{C}$ for $1 \mathrm{~h}$. Then, rescued recombinant phages were added to microplate wells that containing $\mathrm{S}$ and RBD protein and incubated for $1 \mathrm{~h}$ at $37^{\circ} \mathrm{C}$. After being washed, the retained phages were eluted with Glycine$\mathrm{HCl}$ buffer ( $\mathrm{pH}$ 3.0) and neutralized immediately with Tris- $\mathrm{HCl}$ buffer ( $\mathrm{pH} 8.5$ ) to a neutral condition. Next, the eluted phages were used to infect $E$. coli TG1 cells and

\section{Table 1 Primer sequences of amplification VHH genes for recombinant plasmids pcDNA3.1-Nbs-hFc construction}

\begin{tabular}{ll}
\hline Primer & Sequences \\
\hline Nbs-F & 5'-CAGGGATCCCAGGTGCAGCTGGTGGAGTC-3' $^{\prime}$ \\
Nbs-R & 5'-CGCTCGAGTGAGGAGACGGTGACCTGGG-3' $^{\prime}$ \\
biNbs-R1 & $5^{\prime}$-TGAACCGCCTCCACCGCTGCCGCCTCCGCCTGAGGAGACGGTGACCTGGG-3' \\
biNbs-F2 & $5^{\prime}$-GGTGGAGGCGGTTCAGGAGGTGGCGGATCTCAGGTGCAGCTGGTGGAGTC-3' \\
triNbs-R32 & 5'-AGATCCGCCACCTCCGCTGCCGCCTCCGCCTGAGGAGACGGTGACCTGGG-3' $^{\prime}$ \\
triNbs-F3 & 5'-GGAGGTGGCGGATCTGGTGGAGGCGGTTCACAGGTGCAGCTGGTGGAGTC-3' $^{\prime}$ \\
\hline
\end{tabular}


amplified overnight at $37^{\circ} \mathrm{C}$ after infecting with $\mathrm{M} 13 \mathrm{KO} 7$ helper phages. Subsequently, the amplified phages were purified using PEG 4,000/ $\mathrm{NaCl}$ precipitation for the next round bio-panning.

After four rounds of screening, the enrichment of $S$ and RBD specific phage particles were calculated with polyclonal phage ELISA, and then 96 clones were picked randomly for monoclonal identification using an indirect ELISA with HRP-conjugated goat anti-M13 IgG antibody (Sino Biological, Beijing, China). Finally, all positive clones were sequenced and grouped based on their complementary determining regions (CDRs) amino acid sequence.

\section{VHH cloning into HEK293FT expression vector}

A pcDNA3.1 expression vector was constructed firstly to express the $\mathrm{S}$ and RBD protein specific nanobodies in HEK293FT cells. In brief, some elements were designed and cloned into commercial pcDNA3.1(+) vector between NheI and $\mathrm{XbaI}$ sites. DNA sequences, including a secreting signal sequence from the human IgGk chain, multiple cloning site (MCS), human IgG1 Fc, and $6 \times$ His tag following a stop coding sequence, were synthesized (GENERAL BIOL, Anhui, China) and cloned into the multiple cloning sites of the commercial vector pcDNA3.1, and named as pcDNA3.1-MCS-hFc. The VHHs encoding sequences were amplified by PCR using the Nbs-F, Nbs-R primers (Table 1) and cloned between the BamHI and XhoI sites in the pcDNA3.1-MCS-hFc vector. The positive recombinant plasmids were confirmed by sequencing.

\section{Generating bivalent and trivalent VHHs for HEK293FT expression}

To generate bivalent and trivalent tail-to-head $\mathrm{VHH}$ constructs, the $\mathrm{VHH}$ sequences were amplified through combination the following primers, Nbs-F, Nbs-R, biNbs-R1, biNbs-F2, triNbs-R32, triNbs-F3 (Table 1), digested with both $\mathrm{BamHI}$ and $\mathrm{XhoI}$ enzymes and ligated into the vector pcDNA3.1-MCS-hFc. The positive plasmids were propagated with E. coli TransStbl3 cells and used to express recombinant $\mathrm{VHH}$ protein by transfection HEK293FT cells.

\section{Expression and purification of nanobodies}

To produce nanobody-hFc recombinant fusion proteins, the vectors containing monovalent, bivalent and trivalent VHHs were transfected into mammalian cell line HEK293FT cells cultured in Freestyle medium (Gibco, USA) using polyetherimide reagent (PEI, Polysciences Inc. Warrington, USA) based on the manufacture's instruction. After 5 days, the medium that containing secreted nanobody-hFc fusion proteins were harvested by centrifugation at $10,000 \times g$ for $20 \mathrm{~min}$ at $4{ }^{\circ} \mathrm{C}$, and then incubated with Ni-NTA 6FF Agarose for purification. The nanobody-hFc recombinant fusion proteins were eluted using elution buffer ( $20 \mathrm{mM}$ Tris, $250 \mathrm{mM} \mathrm{NaCl}$, $250 \mathrm{mM}$ imidazole, $\mathrm{pH}$ 7.8). The expression and purification were verified using SDS-PAGE and subsequent Coomassie Blue staining. Next, purified VHHs were concentrated on filter tubes (Milipore, USA) and the elution buffer containing imidazole was exchanged with PBS (pH 7.4). Finally, purified VHHs were used directly or stored at $-20^{\circ} \mathrm{C}$.

\section{Indirect ELISA assay}

Determination the binding of nanobody-hFc fusion proteins against $\mathrm{S}$ and RBD protein, microtiter plates were coated overnight at $4{ }^{\circ} \mathrm{C}$ with recombinant $\mathrm{S}$ and $\mathrm{RBD}$ protein, respectively. After washed three times with PBS'T, the coated plates were blocked with $5 \%$ skimmed milk in PBS'T. Dilution series (from $10^{2}$ to $10^{-5} \mu \mathrm{g} / \mathrm{mL}$ ) of nanobody-hFc fusion proteins were added to the wells, followed by adding HRP-conjugated goat anti-human IgG (1/2000, Beyotime Biotechnology). After washing, tetramethylbenzidine liquid substrate (TMB, Sigma) was added to the plates and the reaction was stopped with $2 \mathrm{M} \mathrm{H}_{2} \mathrm{SO}_{4}$, and the optical density at $450 \mathrm{~nm}\left(\mathrm{OD}_{450 \mathrm{~nm}}\right)$ was measured using an automatic ELISA microplate reader. The binding ability was determined using fourparameter nonlinear regression curve fit (Graphpad Prism 5.0).

\section{Production of pseudoviruses}

SARS-CoV-2 S pseudovirus production system was developed in our laboratory as previously described [14]. Briefly, a pLenti-EGFP-luciferase expressing plasmid, a plasmid encoding codon optimized SARS-CoV-2 S protein and a gag/pol expression plasmid were co-transfected into HEK293T cells using polyetherimide reagent (PEI). After transfection $6 \mathrm{~h}$, the medium was replaced with fresh DMEM medium supplemented with $10 \%$ FBS. $48 \mathrm{~h}$ post-transfection, the supernatant was harvested that containing SARS-CoV-2 pseudovirus, and the supernatant was filtered through $0.45 \mu \mathrm{m}$-pore cellulose acetate membranes. To increase the titer of pseudovirus, the supernatants were concentrated to $1 \mathrm{~mL}$ by ultracentrifugation and stored at $-80^{\circ} \mathrm{C}$ until use.

\section{Pseudotyped virus neutralization assay}

To determinate the neutralizing activity of SARSCoV-2 spike and RBD-specific nanobodies against SARS-CoV-2 infection, a pseudovirus neutralization assay was performed. HEK293T-ACE2 stable cell lines 
were constructed as previously described [14]. In brief, HEK293T cells were transfected with a lentivirus vector encoding human ACE2 and puromycin selection marker. Cells were selected with puromycin, and puromycinresistant clones were expanded and verified by FACS for ACE2 expression. HEK293T-ACE2 cells were plated into 96-well cell-culture plates with $2 \times 10^{4}$ cells/well and cultured overnight at $37{ }^{\circ} \mathrm{C}$ with $5 \% \mathrm{CO}_{2}$. The pseudovirus was preincubated with tenfold serial dilution of $\mathrm{Nbs}$ at $37{ }^{\circ} \mathrm{C}$ for $1 \mathrm{~h}$ before being added to cells. Pseudovirus in culture media without Nbs were used as negative control. Medium were changed the following day after infection. $48 \mathrm{~h}$ later, EGFP expression was determined by fluorescent microscopy and flow cytometry to evaluate the neutralization ability. While the cells were lysed using lysis reagent (Promega) after washing with PBS, and relative luciferase activity was measured immediately in the Ultra 384 luminometer (Tecan). The relative luminescence signals (RLU) from the negative control wells were normalized and used to calculate neutralization percentage for each concentration.

\section{Statistical analysis}

All statistical analyses were performed using GraphPad Prism version 5.0 (GraphPad Software, San Diego, CA, USA). All presented data were shown as mean $\pm S D$, which contains three replicates. Comparisons among multiple groups were performed using a two-way

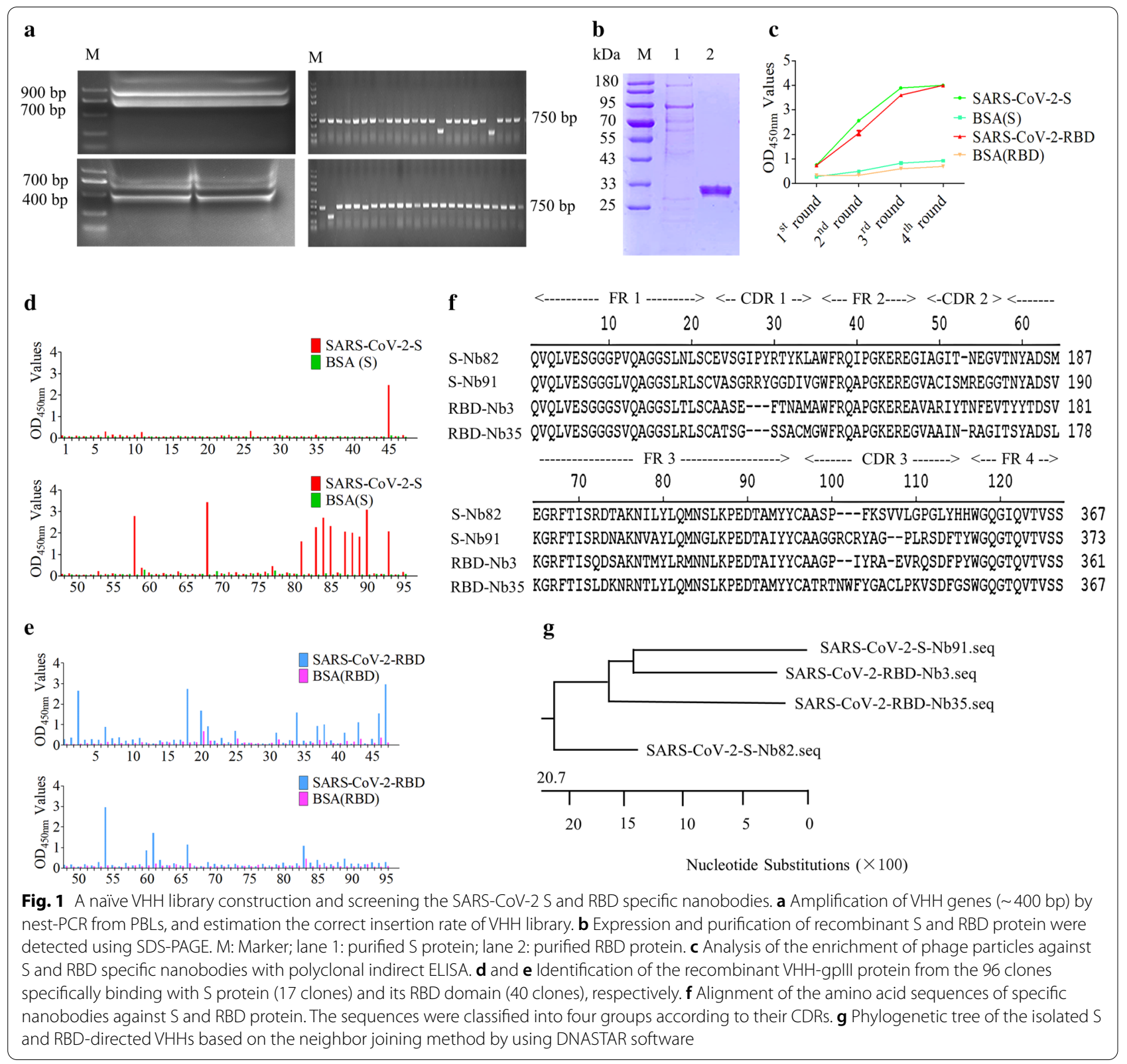


ANOVA test with Bonferroni post-test. $P$-values $<0.05$ were considered statistically significant $\quad\left({ }^{*} P<0.05\right.$; *** $P<0.01 ; * * * P<0.001$ ).

\section{Results}

Construction of the naïve VHH library

Total $4.3 \times 10^{8}$ PBLs were isolated from $200 \mathrm{~mL}$ Bactrian camel blood samples. After reverse-transcription using the extracted RNA as a template, a target band of about 400 bp in size was amplified by nest-PCR (Fig. 1a), and then the PCR fragments and PMECS vectors were digested, ligated, and transformed into TG1 cells. Finally, a phage display naïve VHH library was successfully constructed that containing of approximately $5.7 \times 10^{9}$ individual transformants. Additionally, the insertion rate of VHH genes were evaluated by PCR through randomly picked 48 clones, which was determined to be $93.75 \%$ (Fig. 1a). Subsequently, the sequences of 36 individual clones indicated that the library had good diversity (data not shown).

\section{Preparation of the SARS-CoV-2 recombinant proteins} The recombinant spike (ECD) protein and its RBD domain of SARS-CoV-2 were produced using Baculovirus Expression System. The culture supernatant that containing recombinant $\mathrm{S}$ and RBD proteins was harvested and purified by immobilized metal affinity chromatography (IMAC) using Ni-NTA. The results showed that recombinant $\mathrm{RBD}$ proteins $(27 \mathrm{kDa})$ were expressed successfully and the target was obtained after purification (Fig. 1b), however, there were several bands in the $S$ protein lane (Fig. 1b), and the possible reason is that $S$ protein was cleaved at the $\mathrm{S} 1 / \mathrm{S} 2$ protease cleavage site, in keeping with published data [34-36].

\section{Screening and identification of specific nanobodies against $S$ and RBD protein}

After four rounds bio-panning using purified $S$ and RBD protein, the specific nanobodies particles against $S$ and RBD protein were enriched (Fig. 1c) and the ratio of positive/negative clones $(\mathrm{P} / \mathrm{N})$ increased, from
17.8 to $1.5 \times 10^{3}$, and from 1.5 to $1.8 \times 10^{3}$, respectively (Table 2). The indirect ELISA results revealed that 17 clones (Fig. 1d), and 40 clones (Fig. 1e) could specifically bind with $\mathrm{S}$ and RBD protein, respectively. After the positive clones were sequenced, 4 unique nanobodies were obtained according to the amino acid sequences of the CDRs (Fig. 1f). Multiple sequence alignment and phylogenetic analysis using the neighbor-joining method revealed that 2 unique $\mathrm{S}-\mathrm{Nbs}$ and 2 unique RBDNbs were isolated. The gene evolution and homology between Nb91-hFc and -Nb3-hFc were higher than others (Fig. 1g).

\section{Expression and purification of the nanobodies against $S$ and RBD protein using HEK293FT mammalian system}

The platform of expression nanobody-hFc fusion proteins were constructed successfully based on the commercial pcDNA3.1 vector. In detail, the DNA sequences, including a IgGk singal peptide, MCS, human IgG1 Fc, $6 \times$ His tag, and stop codon were cloned into pcDNA3.1 vector using NheI and XbaI enzymes digestion (Fig. 2a). In addition, a flexible linker was added between Nbs and human IgG1 Fc to increase the flexibility and avoid the formation of higher structures that affect the function of Nbs (Fig. 2a). SDS-PAGE results showed that high purity Nbs-hFc fusions (Nb82-hFc, Nb91-hFc, Nb3-hFc, and $\mathrm{Nb} 35-\mathrm{hFc}$ ) were obtained after purification (Fig. 2b). The indirect ELISA results revealed that: (1) Nb82-hFc could specifically bind with $S$ protein (Fig. 2c), but did not bind with RBD protein (Fig. 2d); (2) Nb91-hFc could bind with $\mathrm{S}$ protein (Fig. 2c), as well as RBD protein (Fig. 2d); (3) $\mathrm{Nb} 3-\mathrm{hFc}$ and Nb35-hFc could recognize RBD protein (Fig. 2d); Nb12-hFc targeting VP2 protein of porcine parvovirus was used as the negative control.

\section{Nanododies neutralize SARS-CoV-2 pseudotyped viruses}

To evaluate the antiviral activity of the $S$ and RBDdirected nanobodies, SARS-CoV-2 $\mathrm{S}$ pseudovirus was used to perform neutralization assays in vitro. The flow cytometry results revealed that the infection efficiency of

Table 2 Enrichment of phage particles against SARS-CoV-2-spike and -RBD specific nanobodies during four rounds of panning

\begin{tabular}{|c|c|c|c|c|c|c|c|c|c|}
\hline \multirow[t]{2}{*}{ Round of screening } & \multirow[t]{2}{*}{ Input (pfu/well) } & \multicolumn{2}{|c|}{ P output (pfu/well) } & \multicolumn{2}{|c|}{$\mathrm{N}$ output (pfu/well) } & \multicolumn{2}{|c|}{ Recovery (P/input) } & \multicolumn{2}{|l|}{$\mathrm{P} / \mathrm{N}$} \\
\hline & & $S$ & RBD & $S$ & RBD & $S$ & RBD & $S$ & RBD \\
\hline 1st round & $1.0 \times 10^{11}$ & $1.5 \times 10^{4}$ & $1.4 \times 10^{4}$ & $8.4 \times 10^{3}$ & $9.1 \times 10^{3}$ & $1.5 \times 10^{-7}$ & $1.4 \times 10^{-7}$ & 17.8 & 1.5 \\
\hline 2nd round & $1.0 \times 10^{11}$ & $6.5 \times 10^{5}$ & $3.4 \times 10^{5}$ & $3.4 \times 10^{3}$ & $2.9 \times 10^{3}$ & $6.5 \times 10^{-6}$ & $3.4 \times 10^{-6}$ & $1.9 \times 10^{2}$ & $1.2 \times 10^{2}$ \\
\hline 3rd round & $1.0 \times 10^{11}$ & $2.6 \times 10^{7}$ & $1.9 \times 10^{7}$ & $4.5 \times 10^{4}$ & $3.8 \times 10^{4}$ & $2.6 \times 10^{-4}$ & $1.9 \times 10^{-4}$ & $5.7 \times 10^{2}$ & $5.0 \times 10^{2}$ \\
\hline 4th round & $1.0 \times 10^{11}$ & $5.4 \times 10^{8}$ & $5.2 \times 10^{8}$ & $3.6 \times 10^{5}$ & $2.7 \times 10^{5}$ & $5.4 \times 10^{-2}$ & $5.2 \times 10^{-2}$ & $1.5 \times 10^{3}$ & $1.8 \times 10^{3}$ \\
\hline
\end{tabular}




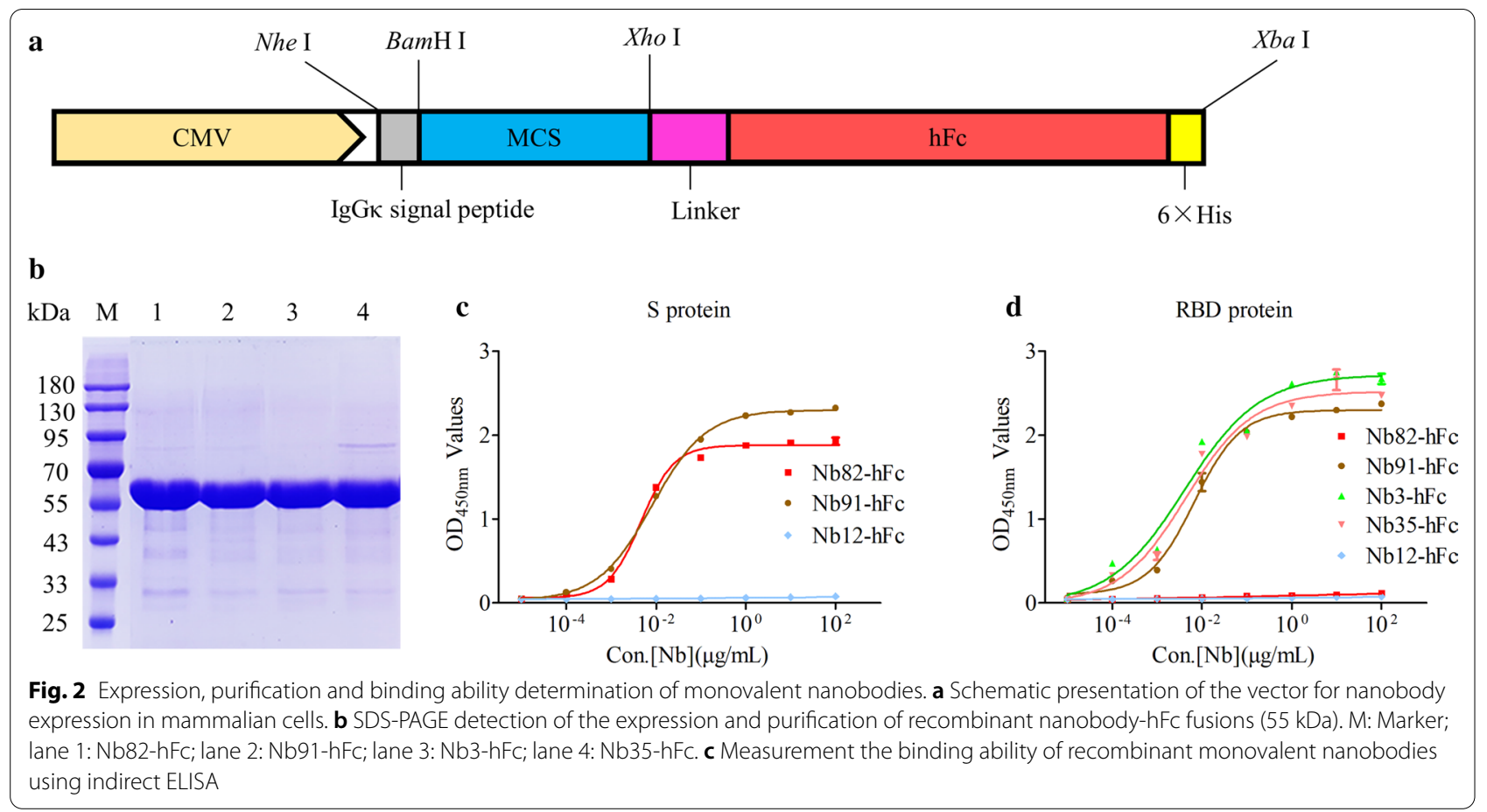

S pseudovirus was approximately $28.35 \%$ (Fig. 3a). The pseudovirus luciferase assay results indicated that Nb91hFc and Nb3-hFc neutralized SARS-CoV-2 pseudotyped virus with an $\mathrm{IC}_{50}$ value at approximately $54.07 \mathrm{nM}$ $(2.65 \mu \mathrm{g} / \mathrm{mL})$ and $32.36 \mathrm{nM}(1.79 \mu \mathrm{g} / \mathrm{mL})$, respectively, whereas Nb82-hFc and Nb35-hFc had no inhibition effect (Fig. 3b). Similar results were obtained from fluorescence and flow cytometry assays (Fig. 3c).

\section{Detection the binding ability of multivalent neutralizing nanobodies}

To further improve the antiviral properties of the neutralizing nanobodies against SARS-CoV-2, multivalent nanobodies were produced. Firstly, homodimer (biNb91hFc, biNb3-hFc), heterodimer (Nb91-Nb3-hFc) and homotrimer (triNb91-hFc, triNb3-hFc) of Nb91 and Nb3 were tandem linked with flexible linker and constructed into pcDNA3.1-MCS-hFc vector (Fig. 4a). The expression procedure of multivalent nanobodies was consistent with monovalent Nbs. After purification, bivalent and trivalent VHHs were obtained from the supernatant of HEK293FT cells (Fig. 4b). The indirect ELISA results indicated that the binding affinity of homodimer and homotrimer with RBD protein were significantly higher than monovalent Nbs $(P<0.001)$ (Fig. 4c and $\mathrm{d})$, and among them, Nb91Nb3-hFc shows the highest affinity $(P<0.001)$ (Fig. $4 d)$.

\section{Neutralization efficiency of the monovalent, bivalent and trivalent nanobodies}

The procedure of multivalent nanobodies pseduovirus neutralization assay were same with monovalent nanobodies. Results showed that the heterodimer nanobody Nb91-Nb3-hFc exhibited the highest neutralizing ability, with an $\mathrm{IC}_{50}$ at $1.54 \mathrm{nM}$ against pseudotyped SARSCoV-2 (Fig. 5a, b). The neutralizing ability of triNb91-hFc $\left(\mathrm{IC}_{50}=4.89 \mathrm{nM}\right)$ improved 11.06 fold compared to the monovalent form $\left(\mathrm{IC}_{50}=54.07 \mathrm{nM}\right)$, and triNb3-hFc $\left(\mathrm{IC}_{50}=4.70 \mathrm{nM}\right)$ improved 6.88 fold than monovalent construct $\left(\mathrm{IC}_{50}=32.36 \mathrm{nM}\right)$ by the pseudovius luciferase assay (Fig. 5a, b). These results revealed that the neutralizing ability of nanobodies could be obviously improved by tandem linking monovalent Nbs (Fig. 5b). Meanwhile, the antiviral activity of multivalent nanobodies were also determined using fluorescent microscopy and flow cytometry assays and similar results were obtained (Fig. 5c).

\section{Discussion}

The COVID-19 pandemic has resulted in an unprecedented world public health crisis. Currently, efficient therapeutics for treatment of COVID-19 are lacking, and the development of a vaccine is likely to take at least 12-18 months [37, 38]. Convalescent plasma is utilized 
to improve therapeutic efficacy in patients with severe COVID-19 because the presence of neutralizing antibodies in the plasma of convalescent patients. Hence, neutralizing antibodies are promising for prevention and therapy of SARS-CoV-2 infection [39, 40]. Although the importance of neutralizing antibodies for protection is indisputable, cross-reactive antibodies or sub-optimal concentration of antibodies can promote pathology, resulting in a phenomenon known as antibody-dependent enhancement (ADE), which has been reported following secondary infections or vaccination with dengue virus and other viruses [41]. According to previous studies, the ADE mechanisms of SARS-CoV and MERS-CoV vaccines are mediated mainly by the engagement of $\mathrm{Fc}$ receptors (FcRs) expressed on macrophages, B cells and monocytes $[42,43]$. So far, the clinical evidence has not shown the COVID-19 vaccines or antibodies to have such an effect. However, given ADE is a theoretical possibility, close monitoring is important to ensure that $\mathrm{ADE}$ can be ruled out as a side effect of COVID-19 vaccines or neutralizing antibodies.
To date, some monoclonal antibodies derived from COVID-19 patients, hybridoma and camelid VHH have been reported with various neutralizing efficiency [4446]. Here, we isolated several nanobodies binding to spike protein and its RBD domain from a naïve $\mathrm{VHH}$ library. Previously, it is reported that multivalent nanobodies formed by tandem linking exhibited stronger binding affinity compare with the monomer nanobody [39, 47]. Consistently, in the present study, we also observed this phenomenon and found the heterodimer nanobody Nb91-Nb3-hFc showed the highest binding affinity and neutralizing activity. Our study indicated that naïve VHH library can be used as a potential resource for rapid acquisition and exploitation of antiviral nanobodies.

Since natural SARS-CoV-2 virus culture and assays must be carried out in a biosafety level-3 laboratory. Therefore, the antiviral activity of nanobodies against authentic viruses in vivo were not performed in the present study. However, compared with the natural viruses, pseudoviruses are well suited for virus entry assays because the pseudotyped particles have similar
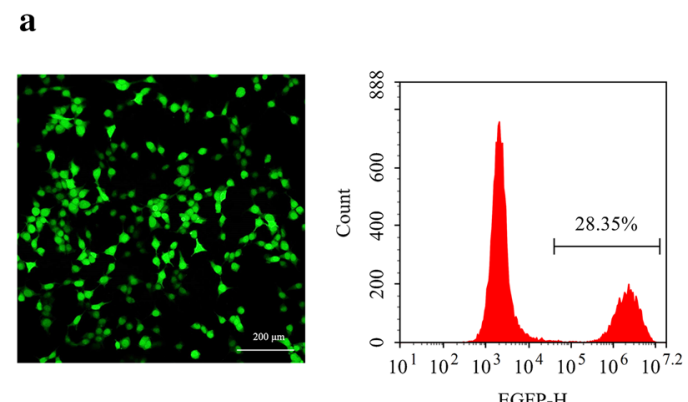

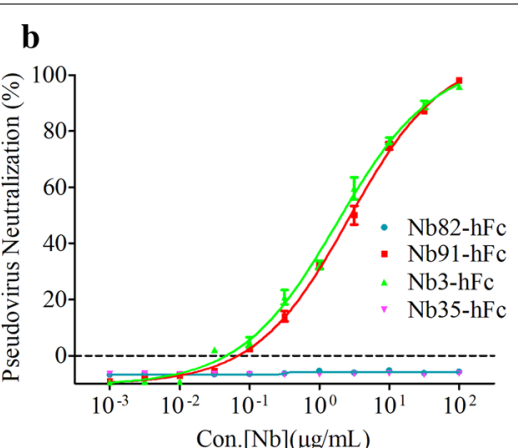

Con. $[\mathrm{Nb}](\mu \mathrm{g} / \mathrm{mL})$

c

$\mathrm{Nb} 82-\mathrm{hFc}$
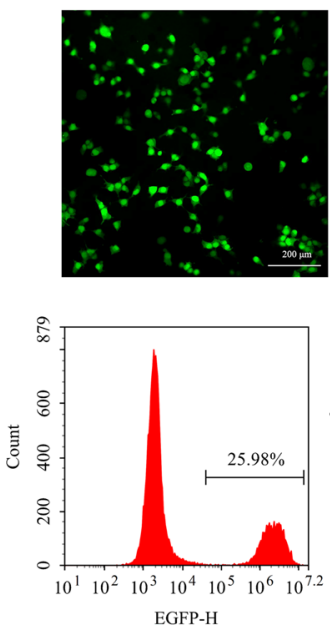

$\mathrm{Nb} 91-\mathrm{hFc}$
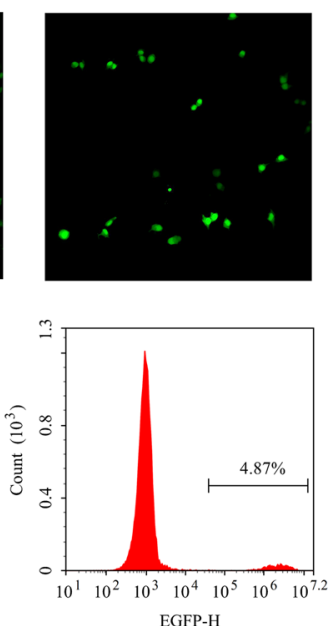

$\mathrm{Nb3}-\mathrm{hFc}$
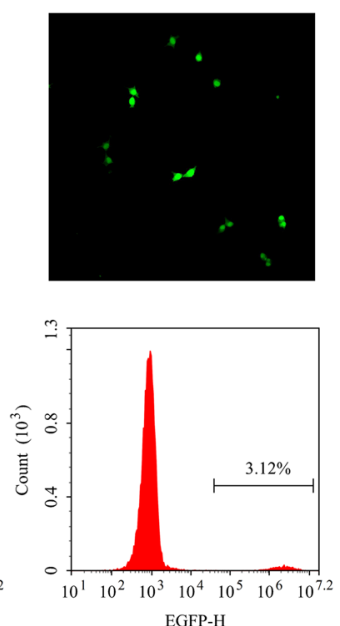

$\mathrm{Nb} 35-\mathrm{hFc}$
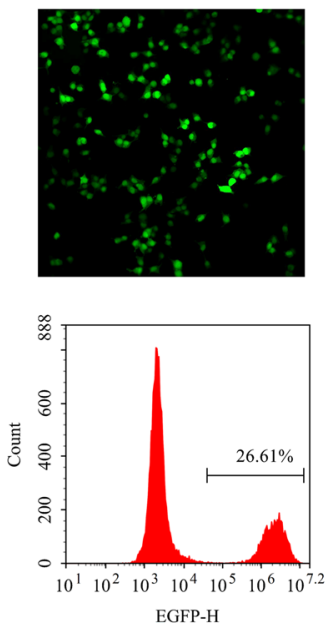

Control (PBS)
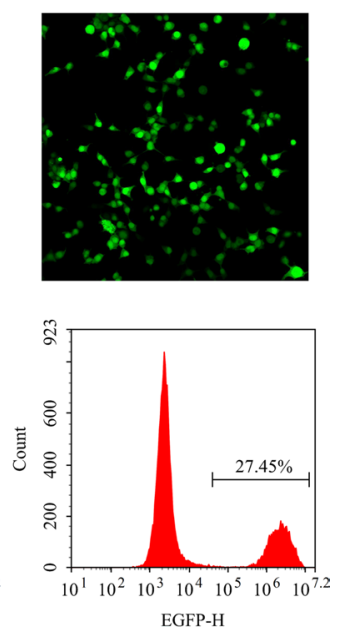

Fig. 3 The selection of the potent neutralizing nanobodies. a Analysis the infection efficiency of pseudovirus with fluorescent microscopy and flow cytometry assays. $\mathbf{b}$ and $\mathbf{c}$ Measurement the neutralization potency of nanobodies with luciferase assay, fluorescent microscopy and flow cytometry assays 
a

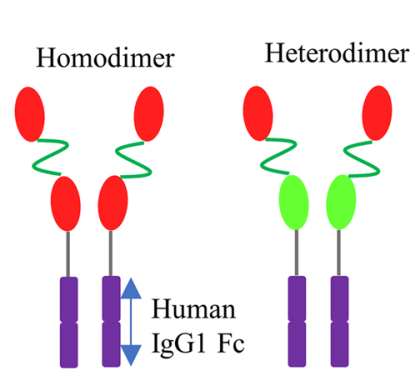

c

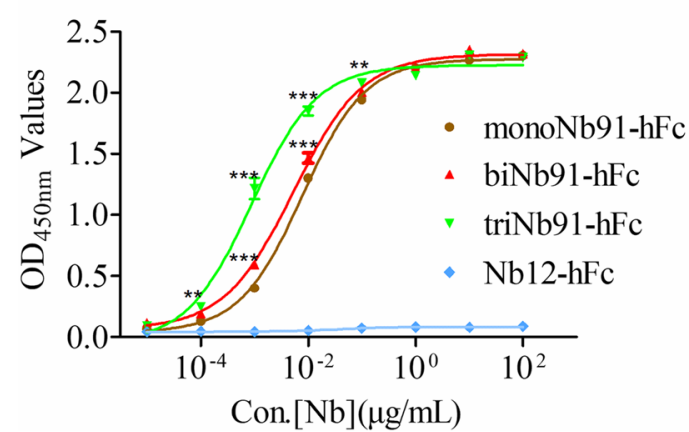

b

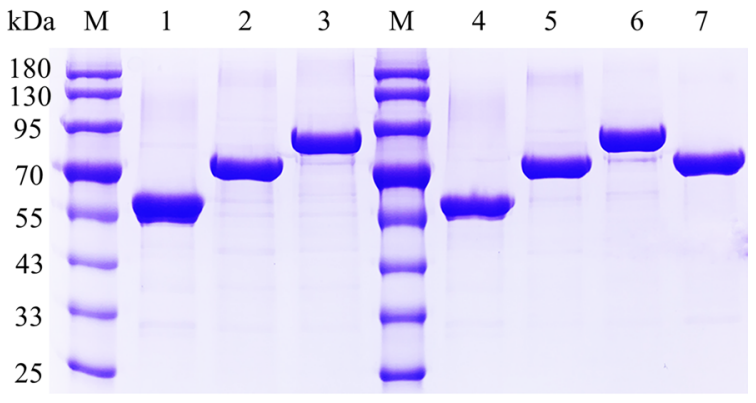

d

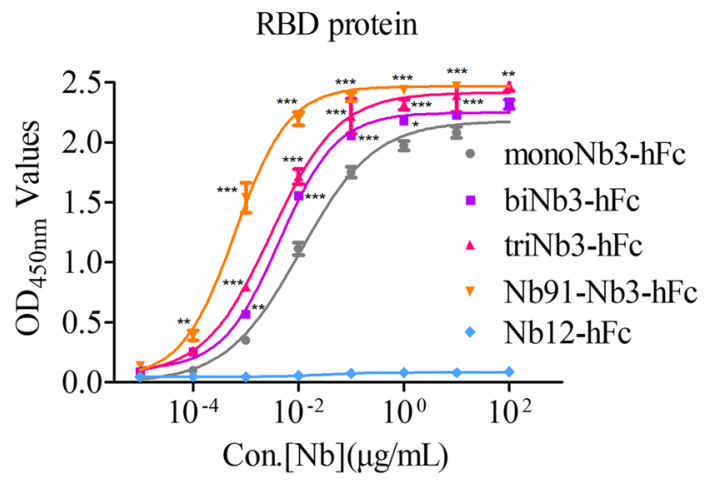

Fig. 4 Development of multivalent nanobodies for highly efficient pseudovirus neutralization. a A schematic view of the multivalent nanobodies design. Homodimer, heterodimer and homotrimer were tandem linked with tail-to-head mode by flexibility linker. b The expression and purification of multivalent nanobodies fusions were analyzed with SDS-PAGE. M: Marker; lane 1 to 7: monoNb91-hFc, biNb91-hFc, triNb91-hFc, monoNb3-hFc, biNb3-hFc, triNb3-hFc and Nb91-Nb3-hFc, respectively. $\mathbf{c}$ and $\mathbf{d}$ Determination the binding affinity of multivalent nanobodies and monovalent Nbs with RBD protein using indirect ELISA

patterners, and can also efficiently simulate the infection process of natural viruses.

Compared with classical monoclonal antibodies, nanobodies show better tissue penetration and extravasation based on the advantage of small size. Recently, caplacizumab, a bivalent nanobody, as the first nanobody drug was approved by the FDA and European Medicines Agency (EMA) for treatment of patients with acquired thrombotic thrombocytopenic purpura [48-50]. Additionally, the lack of post-translational modifications of nanobodies allows it can be expressed in a variety of microbial systems including Escherichia coli, Pichia pastoris and Saccharomyces cerevisiae, which reducing production costs [51, 52]. However, the circulation half-life of nanobodies is significantly shorter in vivo due to their small size, which can be a limitation for disease treatment. Several approaches can be used to extend the half-life of nanobodies in vivo efficiently, such as PEGylation, fusion with Fc, HSA or HSA binding domain. On the other hand, for COVID19 management, nanobodies might be delivered via nasal spray, and in this condition the half-life will be not a problem. For example, ALX-0171, a trivalent nanobody, can reduce the viral load in children with respiratory syncytial virus infection through aerosolized inhalation [53, 54]. Therefore, the neutralizing nanobodies identified in the present study may be exploited to develop aerosolized inhalation products for prevention of COVID-19. 

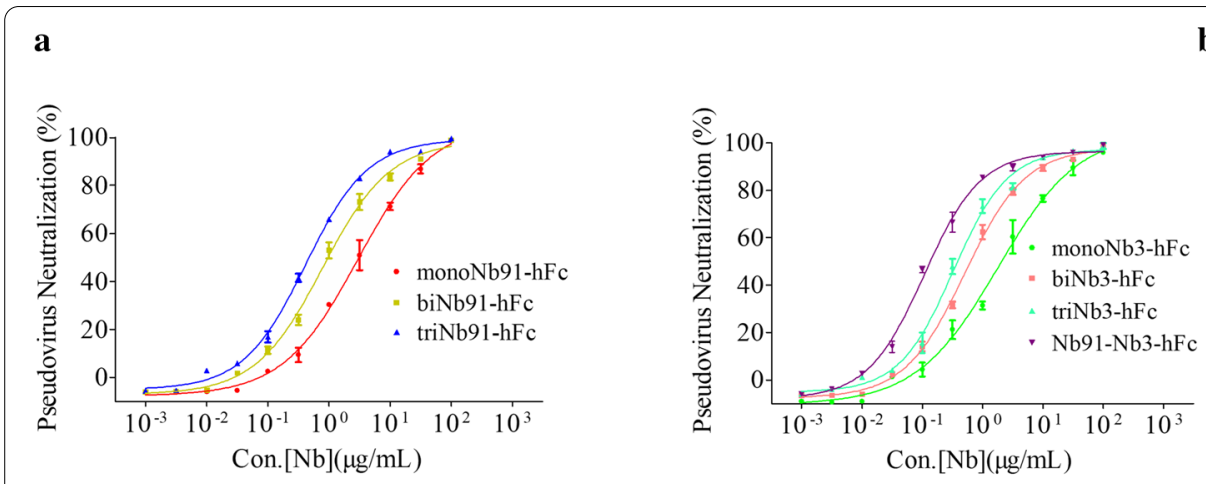

b

Comparison pseudovirus

neutralization potency

\begin{tabular}{cc}
\hline $\mathrm{Nbs}$ & $\mathrm{IC} 50(\mathrm{nM})$ \\
\hline monoNb91-hFc & 54.07 \\
biNb91-hFc & 11.27 \\
triNb91-hFc & 4.89 \\
monoNb3-hFc & 32.36 \\
biNb3-hFc & 6.87 \\
triNb3-hFc & 4.70 \\
Nb91-Nb3-hFc & 1.54 \\
\hline
\end{tabular}

C

biNb91-hFc
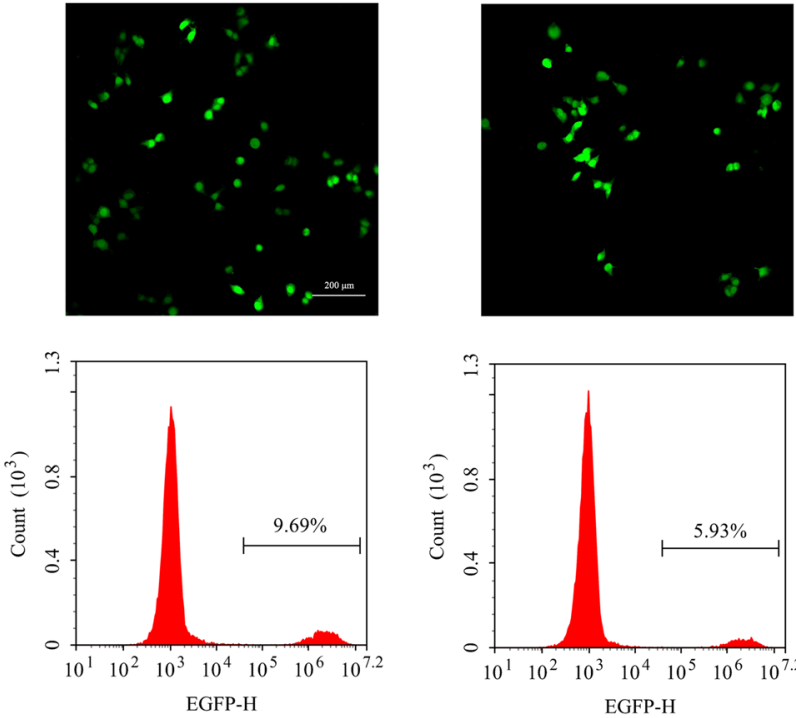

monoNb3-hFc
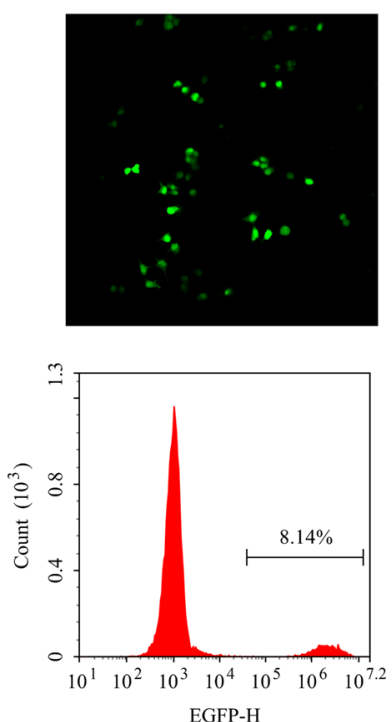

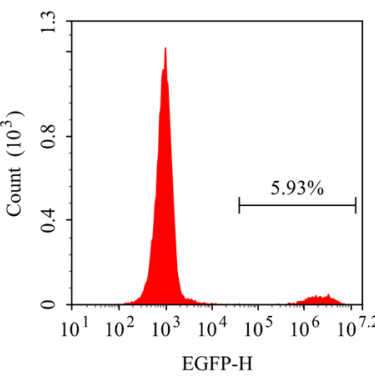

biNb3-hFc
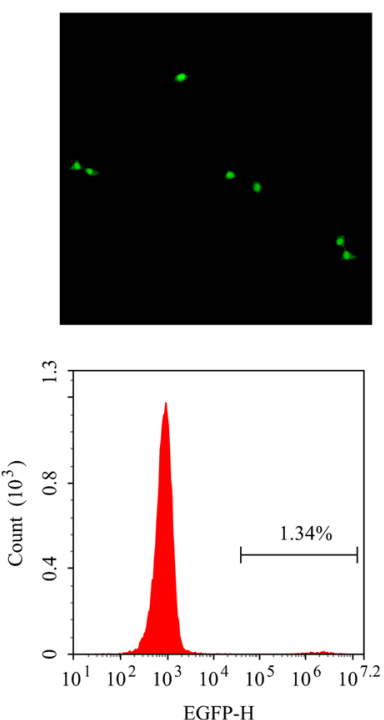

triNb91-hFc
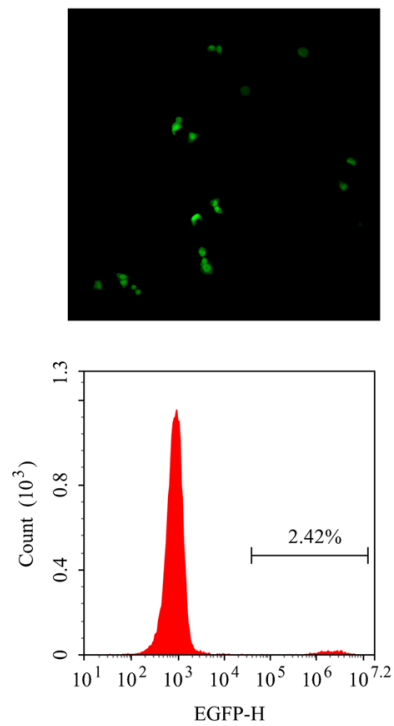

triNb3-hFc
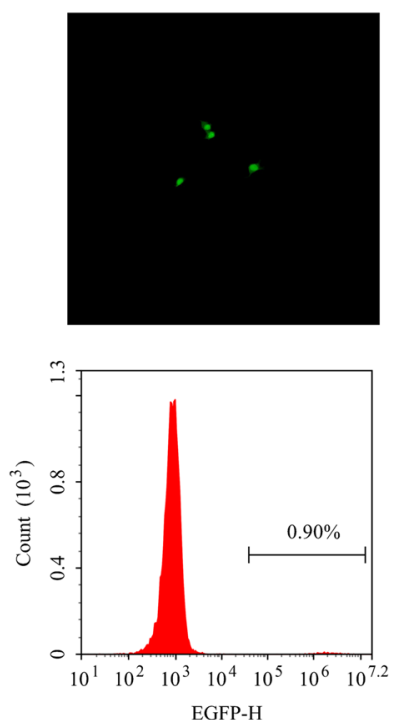

Control (PBS)
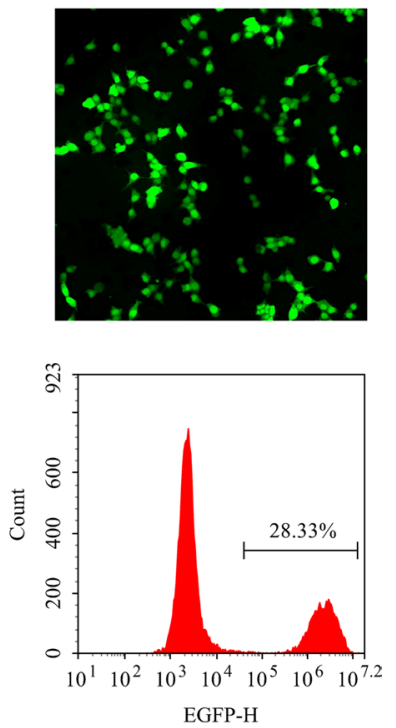

Nb91-Nb3-hFc
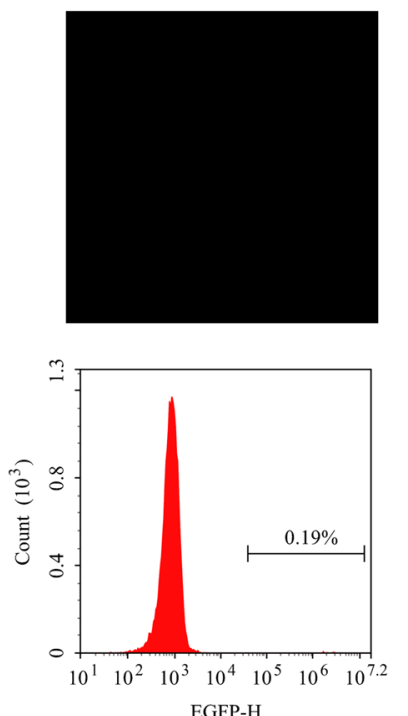

Fig. 5 Evaluation the neutralizing abilities of the potent neutralizing nanobodies. a Neutralization potency was measured by using a pseudotyped virus luciferase neutralization assay. b A table summary of pseudotyped neutralization potency for seven nanobodies. $I C_{50}$ were calculated by fitting a four-parameter logistic curve using Graphpad 5.0. c Neutralization potency of different multivalent nanobodies was performed using fluorescent microscopy and flow cytometry assays 


\section{Conclusions}

In the present work, four specific nanobodies against SARS-CoV-2-S or -RBD protein were screened from a non-immunized Bactrian camel VHH library. Nb91-hFc and Nb3-hFc exhibited highest affinity with RBD protein and neutralizing ability against $S$ pseudovirus. We further compared the neutralizing capacities of tandem linked homo- or hetero-multivalent nanobodies. The results indicated that Nb91-Nb3-hFc possess the most potent neutralizing ability and may serve as an antiviral agent for prevention and treatment of COVID-19.

\section{Acknowledgements}

We thank Hua Huang and Feng Zhou from Renyu biotechnology who contributed to Bactrian camel blood samples collection, isolation the PBLs and phage display library construction.

\section{Authors' contributions}

QZL, ZLZ and APT designed the experiments and drew the figures. QZL, ZLZ and HXL performed library construction, nanobodies screening and neutralizing activity determination. HXL and ZGW participated in nanobodies expression. KHZ participated in camel blood samples collection and PBLs isolation. ZW contributed to pseudovirus preparation. QZ, DHY, SS, NY, QC, MJZ, CL, WHG, HY and CLN contributed to reagents/materials/analysis tools. QZL, ZLZ and HXL analyzed the data. QZL and APT wrote the paper. All authors read and approved the final manuscript.

\section{Funding}

This study was supported by National Natural Science Foundation of China (82073404 \& 81773188), Major Subject of the Science and Technology Department of Sichuan Province (2017SZ0015, 2017SZ0127, 2019YFS0330 and 2019YFS0108) and the special research fund on COVID-19 of West China Hospital Sichuan University (HX-2019-nCoV-064, HX-2019-nCoV-058 \& HX-2019-nCoV-040)

\section{Availability of data and materials}

All data generated or analyzed during this study are included in the article.

\section{Competing interests}

The authors declare that they have no competing interests.

\section{Consent for publication}

Not applicable.

\section{Author details}

${ }^{1}$ State Key Laboratory of Biotherapy and Cancer Center, West China Hospital, Sichuan University, Chengdu 610041, China. ${ }^{2}$ Department of Preventive Veterinary Medicine, College of Veterinary Medicine, Northwest A\&F University, Yangling 712100, China. ${ }^{3}$ College of Veterinary Medicine, Shaanxi Center of Stem Cells Engineering and Technology, Northwest A\&F University, Yangling 712100, China. ${ }^{4}$ Department of Otolaryngology, Head and Neck Surgery, West China Hospital, Sichuan University, Chengdu 610041, China. ${ }^{5}$ Department of Orthopaedics, West China Hospital, Sichuan University, Chengdu 610041, China.

Received: 4 November 2020 Accepted: 6 January 2021

Published online: 29 January 2021

\section{References}

1. Li Q, Guan X, Wu P, Wang X, Zhou L, Tong Y, Ren R, Leung KSM, Lau EHY, Wong JY, et al. Early transmission dynamics in Wuhan, China, of novel coronavirus-infected pneumonia. N Engl J Med. 2020;382:1199-207.

2. Thi Nhu Thao T, Labroussaa F, Ebert N, V'kovski P, Stalder H, Portmann J, Kelly J, Steiner S, Holwerda M, Kratzel A, et al. Rapid reconstruction of SARS-CoV-2 using a synthetic genomics platform. Nature. 2020;582:561-5.
3. Bedford J, Enria D, Giesecke J, Heymann DL, Ihekweazu C, Kobinger G, Lane HC, Memish Z, Oh M-D, Sall AA, et al. COVID-19: towards controlling of a pandemic. Lancet. 2020;395:1015-8.

4. Callaway E, Cyranoski D, Mallapaty S, Stoye E, Tollefson J. The coronavirus pandemic in five powerful charts. Nature. 2020;579:482-3.

5. Zhou P, Yang XL, Wang XG, Hu B, Zhang L, Zhang W, Si HR, Zhu Y, Li B, Huang $\mathrm{CL}$, et al. A pneumonia outbreak associated with a new coronavirus of probable bat origin. Nature. 2020;579:270-3.

6. Peng X, Xu X, Li Y, Cheng L, Zhou X, Ren B. Transmission routes of 2019nCoV and controls in dental practice. Int J Oral Sci. 2020;12:9.

7. Ludwig S, Zarbock A. Coronaviruses and SARS-CoV-2: a brief overview. Anesth Analg. 2020;131:93-6.

8. Wu F, Zhao S, Yu B, Chen YM, Wang W, Song ZG, Hu Y, Tao ZW, Tian JH, Pei $Y Y$, et al. A new coronavirus associated with human respiratory disease in China. Nature. 2020;579:265-9.

9. Bangaru S, Ozorowski G, Turner HL, Antanasijevic A, Huang D, Wang X, Torres JL, Diedrich JK, Tian JH, Portnoff AD, et al. Structural analysis of fulllength SARS-CoV-2 spike protein from an advanced vaccine candidate. Science. 2020;370:1089-94.

10. Lan J, Ge J, Yu J, Shan S, Zhou H, Fan S, Zhang Q, Shi X, Wang Q, Zhang $L$, Wang $X$. Structure of the SARS-CoV-2 spike receptor-binding domain bound to the ACE2 receptor. Nature. 2020;581:215-20.

11. Sternberg A, Naujokat C. Structural features of coronavirus SARS-CoV-2 spike protein: targets for vaccination. Life Sci. 2020;257:118056.

12. Wrapp D, Wang N, Corbett KS, Goldsmith JA, Hsieh CL, Abiona O, Graham BS, McLellan JS. Cryo-EM structure of the 2019-nCoV spike in the prefusion conformation. Science. 2020;367:1260-3.

13. Quinlan B, Mou H, Zhang L, Guo Y, He W, Ojha A, Parcells M, Luo G, Li W, Zhong G, et al. The SARS-CoV-2 Receptor-Binding Domain Elicits a Potent Neutralizing Response Without Antibody-Dependent Enhancement. SSRN Electron J. 2020. https://doi.org/10.1101/2020.04.10.036418.

14. Yang J, Wang W, Chen Z, Lu S, Yang F, Bi Z, Bao L, Mo F, Li X, Huang Y, et al. A vaccine targeting the RBD of the $S$ protein of SARS-CoV-2 induces protective immunity. Nature. 2020;586:572-7.

15. Conrath KE, Wernery U, Muyldermans S, Nguyen VK. Emergence and evolution of functional heavy-chain antibodies in Camelidae. Dev Comp Immunol. 2003;27:87-103.

16. Hamers-Casterman C, Atarhouch T, Muyldermans S, Robinson G, Hamers C, Songa EB, Bendahman N, Hamers R. Naturally occurring antibodies devoid of light chains. Nature. 1993;363:446-8.

17. Muyldermans S, Atarhouch T, Saldanha J, Barbosa JA, Hamers R. Sequence and structure of VH domain from naturally occurring camel heavy chain immunoglobulins lacking light chains. Protein Eng. 1994;7:1129-35.

18. Riechmann L, Muyldermans S. Single domain antibodies: comparison of camel VH and camelised human VH domains. J Immunol Methods. 1999;231:25-38.

19. Bannas P, Hambach J, Koch-Nolte F. Nanobodies and nanobody-based human heavy chain antibodies as antitumor therapeutics. Front Immunol. 2017:8:1603.

20. de Bruin RCG, Veluchamy JP, Lougheed SM, Schneiders FL, Lopez-Lastra S, Lameris R, Stam AG, Sebestyen Z, Kuball J, Molthoff CFM, et al. A bispecific nanobody approach to leverage the potent and widely applicable tumor cytolytic capacity of Vy9V82-T cells. Oncoimmunology. 2017;7:e1375641.

21. Kijanka M, Dorresteijn B, Oliveira S, van Bergen en Henegouwen PM. Nanobody-based cancer therapy of solid tumors. Nanomedicine (Lond). 2015;10:161-74.

22. Laursen NS, Friesen RHE, Zhu X, Jongeneelen M, Blokland S, Vermond J, van Eijgen A, Tang C, van Diepen H, Obmolova G, et al. Universal protection against influenza infection by a multidomain antibody to influenza hemagglutinin. Science. 2018;362:598-602.

23. Steeland S, Vandenbroucke RE, Libert C. Nanobodies as therapeutics: big opportunities for small antibodies. Drug Discov Today. 2016;21:1076-113.

24. Zhao G, He L, Sun S, Qiu H, Tai W, Chen J, Li J, Chen Y, Guo Y, Wang Y, et al. A novel nanobody targeting middle east respiratory syndrome coronavirus (MERS-CoV) receptor-binding domain has potent crossneutralizing activity and protective efficacy against MERS-CoV. J Virol. 2018:92:e00837-18.

25. Hanke L, Knockenhauer KE, Brewer RC, van Diest E, Schmidt Fl, Schwartz TU, Ploegh HL. The antiviral mechanism of an influenza A virus nucleoprotein-specific single-domain antibody fragment. mBio. 2016;7:e01569-16. 
26. Van Hout A, Klarenbeek A, Bobkov V, Doijen J, Arimont M, Zhao C, Heukers R, Rimkunas R, de Graaf C, Verrips T, et al. CXCR4-targeting nanobodies differentially inhibit CXCR4 function and HIV entry. Biochem Pharmacol. 2018;158:402-12.

27. Wu X, Li Y, Huang B, Ma X, Zhu L, Zheng N, Xu S, Nawaz W, Xu C, Wu Z. A single-domain antibody inhibits SFTSV and mitigates virus-induced pathogenesis in vivo. JCl Insight. 2020;5:e136855.

28. Lu G, Hu Y, Wang Q, Qi J, Gao F, Li Y, Zhang Y, Zhang W, Yuan Y, Bao J, et al. Molecular basis of binding between novel human coronavirus MERS-CoV and its receptor CD26. Nature. 2013;500:227-31.

29. Liu H, Wang Y, Duan H, Zhang A, Liang C, Gao J, Zhang C, Huang B, Li Q, Li N, et al. An intracellularly expressed Nsp9-specific nanobody in MARC145 cells inhibits porcine reproductive and respiratory syndrome virus replication. Vet Microbiol. 2015;181:252-60.

30. Lu Q, Li X, Zhao J, Zhu J, Luo Y, Duan H, Ji P, Wang K, Liu B, Wang X, et al. Nanobody-horseradish peroxidase and -EGFP fusions as reagents to detect porcine parvovirus in the immunoassays. J Nanobiotechnol. 2020;18:7.

31. Sheng Y, Wang K, Lu Q, Ji P, Liu B, Zhu J, Liu Q, Sun Y, Zhang J, Zhou EM, Zhao Q. Nanobody-horseradish peroxidase fusion protein as an ultrasensitive probe to detect antibodies against Newcastle disease virus in the immunoassay. J Nanobiotechnol. 2019;17:35.

32. Vincke C, Gutiérrez C, Wernery U, Devoogdt N, Hassanzadeh-Ghassabeh $G$, Muyldermans S. Generation of single domain antibody fragments derived from camelids and generation of manifold constructs. Methods Mol Biol. 2012;907:145-76.

33. Ji P, Zhu J, Li X, Fan W, Liu Q, Wang K, Zhao J, Sun Y, Liu B, Zhou EM, Zhao Q. Fenobody and RANbody-based sandwich enzyme-linked immunosorbent assay to detect Newcastle disease virus. J Nanobiotechnol. 2020;18:44.

34. Cai Y, Zhang J, Xiao T, Peng H, Sterling SM, Walsh RM Jr, Rawson S, RitsVolloch S, Chen B. Distinct conformational states of SARS-CoV-2 spike protein. Science. 2020;369:1586-92.

35. Hoffmann M, Kleine-Weber H, Pöhlmann S. A multibasic cleavage site in the spike protein of SARS-CoV-2 is essential for infection of human lung cells. Mol Cell. 2020;78:779-84.e775.

36. Hoffmann M, Kleine-Weber $\mathrm{H}$, Schroeder $\mathrm{S}$, Krüger N, Herrler T, Erichsen S, Schiergens TS, Herrler G, Wu NH, Nitsche A, et al. SARS-CoV-2 cell entry depends on ACE2 and TMPRSS2 and is blocked by a clinically proven protease inhibitor. Cell. 2020;181:271-80.e278.

37. Riva L, Yuan S, Yin X, Martin-Sancho L, Matsunaga N, Pache L, BurgstallerMuehlbacher S, De Jesus PD, Teriete P, Hull MV, et al. Discovery of SARS-CoV-2 antiviral drugs through large-scale compound repurposing. Nature. 2020;586:113-9.

38. Wang C, Li W, Drabek D, Okba NMA, van Haperen R, Osterhaus A, van Kuppeveld FJM, Haagmans BL, Grosveld F, Bosch BJ. A human monoclonal antibody blocking SARS-CoV-2 infection. Nat Commun. 2020;1 1:2251.

39. Li W, Schäfer A, Kulkarni SS, Liu X, Martinez DR, Chen C, Sun Z, Leist SR, Drelich A, Zhang L, et al. High potency of a bivalent human $\mathrm{V}(\mathrm{H})$ domain in SARS-CoV-2 animal models. Cell. 2020;183:429-41.e416.

40. Ouyang J, Isnard S, Lin J, Fombuena B, Peng X, Routy JP, Chen Y. Convalescent plasma: the relay baton in the race for coronavirus disease 2019 treatment. Front Immunol. 2020;11:570063.

41. Lee WS, Wheatley AK, Kent SJ, DeKosky BJ. Antibody-dependent enhancement and SARS-CoV-2 vaccines and therapies. Nat Microbiol. 2020;5:1185-91.
42. Wang SF, Tseng SP, Yen CH, Yang JY, Tsao CH, Shen CW, Chen KH, Liu FT, Liu WT, Chen YM, Huang JC. Antibody-dependent SARS coronavirus infection is mediated by antibodies against spike proteins. Biochem Biophys Res Commun. 2014;451:208-14.

43. Wen J, Cheng Y, Ling R, Dai Y, Huang B, Huang W, Zhang S, Jiang Y. Antibody-dependent enhancement of coronavirus. Int J Infect Dis. 2020;100:483-9.

44. Shi R, Shan C, Duan X, Chen Z, Liu P, Song J, Song T, Bi X, Han C, Wu L, et al. A human neutralizing antibody targets the receptor-binding site of SARS-CoV-2. Nature. 2020;584:120-4.

45. Sun Z, Chen C, Li W, Martinez DR, Drelich A, Baek DS, Liu X, Mellors JW Tseng CT, Baric RS, Dimitrov DS. Potent neutralization of SARS-CoV-2 by human antibody heavy-chain variable domains isolated from a large library with a new stable scaffold. MAbs. 2020;12:1778435.

46. Wrapp D, De Vlieger D, Corbett KS, Torres GM, Wang N, Van Breedam W, Roose K, van Schie L, Hoffmann M, Pöhlmann S, et al. Structural basis for potent neutralization of betacoronaviruses by single-domain camelid antibodies. Cell. 2020;181:1004-15.e1015.

47. Xiang Y, Nambulli S, Xiao Z, Liu H, Sang Z, Duprex W, Schneidman D, Zhang C, Shi Y. Versatile, multivalent nanobody cocktails efficiently neutralize SARS-CoV-2. Science. 2020. https://doi. org/10.1101/2020.08.24.264333

48. Elverdi T, Eskazan AE. Caplacizumab as an emerging treatment option for acquired thrombotic thrombocytopenic purpura. Drug Des Devel Ther. 2019;13:1251-8.

49. Sargentini-Maier ML, De Decker P, Tersteeg C, Canvin J, Callewaert F, De Winter $\mathrm{H}$. Clinical pharmacology of caplacizumab for the treatment of patients with acquired thrombotic thrombocytopenic purpura. Expert Rev Clin Pharmacol. 2019;12:537-45.

50. Scully M, Cataland SR, Peyvandi F, Coppo P, Knöbl P, Kremer Hovinga JA, Metjian A, de la Rubia J, Pavenski K, Callewaert F, et al. Caplacizumab treatment for acquired thrombotic thrombocytopenic purpura. N Engl J Med. 2019;380:335-46.

51. Jovčevska I, Muyldermans S. The therapeutic potential of nanobodies. BioDrugs. 2020;34:11-26.

52. Kolkman JA, Law DA. Nanobodies_from llamas to therapeutic proteins. Drug Discov Today Technol. 2010;7:e139-46.

53. Cunningham S, Piedra PA, Martinon-Torres F, Szymanski H, Brackeva B, Dombrecht E, Detalle L, Fleurinck C. Nebulised ALX-0171 for respiratory syncytial virus lower respiratory tract infection in hospitalised children: a double-blind, randomised, placebo-controlled, phase $2 \mathrm{~b}$ trial. Lancet Respir Med. 2021;9:21-32.

54. Detalle L, Stohr T, Palomo C, Piedra PA, Gilbert BE, Mas V, Millar A, Power UF, Stortelers C, Allosery K, et al. Generation and characterization of ALX-0171, a potent novel therapeutic nanobody for the treatment of respiratory syncytial virus infection. Antimicrob Agents Chemother. 2016;60:6-13.

\section{Publisher's note}

Springer Nature remains neutral with regard to jurisdictional claims in published maps and institutional affiliations.

Ready to submit your research? Choose BMC and benefit from:

- fast, convenient online submission

- thorough peer review by experienced researchers in your field

- rapid publication on acceptance

- support for research data, including large and complex data types

- gold Open Access which fosters wider collaboration and increased citations

- maximum visibility for your research: over 100M website views per year

At BMC, research is always in progress.

Learn more biomedcentral.com/submissions 